\title{
Rehearsal in serial memory for visual-spatial information: Evidence from eye movements
}

\author{
SÉBASTIEN TREMBLAY \\ Université Laval, Quebec, Quebec, Canada \\ and \\ JEAN SAINT-AUBIN and ANNIE JALBERT \\ Université de Moncton, Moncton, New Brunswick, Canada
}

\begin{abstract}
It is well established that rote rehearsal plays a key role in serial memory for lists of verbal items. Although a great deal of research has informed us about the nature of verbal rehearsal, much less attention has been devoted to rehearsal in serial memory for visual-spatial information. By using the dot task - a visual-spatial analogue of the classical verbal serial recall task-with delayed recall, performance and eyetracking data were recorded in order to establish whether visual-spatial rehearsal could be evidenced by eye movement. The use of eye movement as a form of rehearsal is detectable (Experiment 1), and it seems to contribute to serial memory performance over and above rehearsal based on shifts of spatial attention (Experiments 1 and 2).
\end{abstract}

In the last 4 decades, the capacity to remember the order of events over the short term has been extensively investigated; however, most of the research has been devoted to the study of verbal serial memory. There is a substantial body of work on the nature of verbal rehearsal and its contribution to serial memory for lists of verbal items (see, e.g., Longoni, Richardson, \& Aiello, 1993). It is widely assumed that some form of rehearsal contributes to memory; many researchers view rehearsal as a speech-based or articulatory system involved in recycling representations over time (e.g., Baddeley, Thomson, \& Buchanan, 1975; Schweickert \& Boruff, 1986), whereas others conceptualize rehearsal as a mechanism - cyclic reinsantiation - that produces internal copies of to-be-remembered stimuli (e.g., Farrell \& Lewandowsky, 2002; Nairne, 2002). There is ample evidence that rehearsal plays a role in serial memory of verbal material, but it is still unclear whether there is a similar mechanism in serial memory for visual-spatial information (see, e.g., Awh \& Jonides, 2001; Baddeley, 1986). Empirical work directed at examining rehearsal mechanisms in the spatial domain is still at an early stage. The purpose of the present experiments was twofold: to further examine whether there is some form of visual-

This research was supported by operating grants from the Natural Sciences and Engineering Research Council of Canada to S.T. and to J.S.-A. Also, this collaborative work received support from the Québec and New Brunswick governments through the Cooperation in Advanced Education and Research program. Thanks are due Isabel Savoie and Julie Latulippe for running the experiments. We also thank Robert Hughes and Katherine Guérard for critical reading of an earlier draft. Correspondence can be addressed to S. Tremblay, École de psychologie, Université Laval, Quebec, PQ, G1K 7P4 Canada (e-mail: sebastien.tremblay@psy .ulaval.ca orsaint-aj@umoncton.ca). spatial rehearsal and to establish whether eye movement is a good candidate for such a mechanism.

Some researchers have suggested that rehearsal for visual-spatial information is based on eye movement (e.g., Baddeley, 1986), and others that the active system responsible for spatial rehearsal is also involved in the planning and production of body movement (including the eyes; see, e.g., Logie, 1995), whereas still others have proposed that spatial-attentional mechanisms - shifts of spatial selective attention - are involved in the maintenance of visual-spatial information (e.g., Awh \& Jonides, 2001; Smyth, 1996). Awh and his collaborators (Awh \& Jonides, 2001; see also Postle, Awh, Jonides, Smith, \& D'Esposito, 2004) have argued that rehearsal for visualspatial information involves shifts of selective attention to the location of interest. Temporary maintenance of a single location is impaired if attention is attracted away from that location during a short retention interval (e.g., Awh, Jonides, \& Reuter-Lorenz, 1998). The entire line of research carried out by Awh and his collaborators employed short-term memory (STM) tasks that required recognition for a single item. Their interest was centered on the temporary retention of item information, rather than on serial memory, whereas most, if not all, of the theoretical development on verbal rehearsal has been based on serial memory. Lawrence, Myerson, and Abrams (2004) suggested that the mechanisms of spatial rehearsal involved in serial memory might be different from those involved in the short-term retention of a single item (see also Zimmer, Speiser, \& Seidler, 2003).

According to Logie (1995), active spatially based rehearsal is closely linked to the planning of movement. The latter claim originates mainly from demonstrations of concurrent conflicts between memory tasks involving 
visual-spatial information and different forms of taskirrelevant movement, such as sequential tapping (e.g., Farmer, Berman, \& Fletcher, 1986; Meiser \& Klauer, 1999), arm movement (e.g., Smyth \& Scholey, 1994), and also eye movement (e.g., Lawrence, Myerson, Oonk, \& Abrams, 2001). However, it could be argued that the disruptive effects of body movements on the maintenance of spatial information can be attributed to the spatial shifts of attention that accompany movements, rather than to their planning (e.g., Smyth, 1996).

In the case of eye movement, Pearson and Sahraie (2003), using a computerized version of the Corsi block with delayed recall, demonstrated that concurrent eye movements interfere with spatial span to a greater extent than do shifts of spatial attention with the eyes fixated (see also Lawrence et al., 2004). This finding could be taken to suggest that eye movement is involved in visual-spatial rehearsal, given that its impact on recall is independent of shifts in spatial selective attention. However, the same authors did not detect any pattern of eye movement during a 5 -sec retention interval. A criticism of the Corsi block task is that the use of a fixed set of items, combined with that of grids, may encourage the formation of a representation in long-term memory of the pattern as a whole and, thus, promote visual imagery, rather than overt rehearsal based on eye movement. Indeed, it is possible for to-be-remembered (TBR) visual-spatial items to be maintained as a visual pattern, with little involvement of serial memory (see Smyth \& Scholey, 1994). So far, there is no direct evidence as to whether, as Baddeley (1986) initially suggested, some form of visual-spatial rehearsal contributes appreciably to serial memory performance and whether such rehearsal would rely on eye movement. The possible link between serial memory and rehearsal based on eye movement is consistent with the finding that a serial pattern of fixations on the objects of a scene leads to typical serial memory performance, with strong primacy and recency effects (Zelinsky \& Loschky, 2005).

All the evidence reported so far is based on performance data in the experimental context of dual tasks in which STM for visual-spatial information is disrupted by a concurrent task, such as irrelevant eye movement (e.g., Lawrence et al., 2004). In the present study, we adopted a novel approach in which eye movements, as well as performance data, were recorded for the visual-spatial serial memory task and, thus, provided a direct measure of rehearsal based on eye movement. The latter task was a visual-spatial version of the immediate serial recall task that had previously been employed (see Jones, Farrand, Stuart, \& Morris, 1995). Another key aspect of the present series of experiments is that we report serial position data, in an attempt to examine whether there was a relationship between visual-spatial rehearsal and the pattern of errors.

\section{EXPERIMENT 1}

In the first experiment, we attempted to detect a pattern of eye movements in relation to recall performance that could indicate their contribution to visual-spatial rehearsal. As in Pearson and Sahraie (2003), following presentation of the TBR dots, there was a retention interval - this time, $10 \mathrm{sec}$. During that interval, the participants were required to keep looking at the screen but were free to move their eyes.

\section{Method}

Participants. Twenty-four unpaid undergraduate students (14 women) from Université de Moncton volunteered to participate in this experiment. All the participants had normal or corrected-tonormal vision.

Materials. Seven dots $1 \mathrm{~cm}$ in diameter were presented in different locations on a computer screen, one dot at a time, within a $17 \times$ $17 \mathrm{~cm}$ white space at the center of the computer screen. In addition, the space outside the critical area was also white; that is, there was no border around the critical area, making delimitation of the area on the basis of external cues impossible. The coordinates for the center of the dots were randomly generated, with the constraint that the centers of all the dots, taken two by two, were separated by at least $2 \mathrm{~cm}$ and, at most, $10 \mathrm{~cm}$. On each trial, the dots were displayed at a rate of one every second ( $700 \mathrm{msec}$ on, $300 \mathrm{msec}$ off).

Apparatus. Eye movements were measured with an SR Research Ltd EyeLink II system. This system has high accuracy $\left(<0.5^{\circ}\right.$ on average) and a high sampling rate $(500 \mathrm{~Hz})$. The EyeLink II headband has three cameras, allowing simultaneous tracking both of eyes and of head position for head motion compensation. Only the pupil of the participant's dominant eye was tracked in our study. In the present investigation, the configurable acceleration and velocity thresholds were set to detect saccades of $0.5^{\circ}$ or greater.

Stimulus displays were presented on two monitors, one for the participant and the other for the experimenter. The experimenter's monitor was used to give feedback to the experimenter in real time about the participants' computed gaze positions. This allowed the experimenter to evaluate system accuracy and to initiate a recalibration, if necessary.

Procedure. The participants took part individually in one session lasting approximately $25 \mathrm{~min}$. The first $5 \mathrm{~min}$ were used to calibrate the eyetracker system, and the last 20 min were used for the memory task. The participants were seated approximately $50 \mathrm{~cm}$ from the computer screen on which the visual-spatial memory task was displayed. The experimenter was present throughout the experiment.

On each trial, a sequence of seven black dots was presented on a computer monitor for serial recall. Each successive dot was presented in a different position on the monitor. After the presentation of the seventh dot, during the 10 -sec retention interval, all seven TBR dots were simultaneously re-presented at the spatial locations at which they had been presented initially, and the dots remained on screen until the end of the recall period. After the end of the retention interval, the experimenter gave the signal to the participants to start responding. The participants performed a reconstruction task; they had to reproduce the order of presentation by pointing a finger at the dots in their presentation order. The experimenter noted the order on answer sheets on which the seven dots were displayed as on the screen. The entire session was recorded on videotape and subsequently used to clarify any ambiguity that could have occurred during the online data scoring. Written instructions encouraged the participants to respond as quickly and accurately as possible and informed them that once an item had been selected, it would not be possible to point to it again. After the initial nine-point calibration procedure, the memory task began. Four practice trials preceded the 24 experimental trials. The experimenter initiated the presentation of a trial when the participant was fixating a single calibration dot at the center of the screen (distinct in size and appearance from the TBR dots). This was done to ensure eye movement recording accuracy. The participants were warned that the calibration dot was not part of the memory task. 


\section{Results}

Eye movement data were scored with the EyeLink Data Viewer program, which displayed the dots that were presented and, superimposed on it, the participants' successive landing positions. Eye movements were scored during the 10-sec retention interval. The amount of rehearsal engaged in on each trial was computed as the number of different adjacent dot pairs rehearsed in the correct order-because it is commonly assumed in the verbal domain that items are rehearsed in the correct order (e.g., Rundus \& Atkinson, 1970). For instance, the participant could fixate in succession the first and the second adjacent dots, and this would count as one rehearsed pair. However, if the participant fixated successively the second dot and then the first, this would not count as one successfully rehearsed pair of dots. With this criterion, there were a maximum of six possible pairs $[1-2,2-3,3-4,4-5,5-6$, and 6-7]. Because the analysis was at the pair level, the overall sequence of events was irrelevant. In other words, on a given trial, a participant who would fixate Item 4 and then Item 5 , followed by a series of erratic fixations and then by a fixation on Item 1 and then on Item 2 , would receive a score of two different item pairs rehearsed in the correct order. It is worth noting that the number of times a specific pair was rehearsed was irrelevant with this criterion: Once a specific pair had been scored as rehearsed, it could not be scored again. ${ }^{1}$

With the number of different pairs rehearsed in the correct order as the criterion, out of the 576 trials ( 24 trials $\times$ 24 participants), no pair was rehearsed on 196 trials, a single pair was rehearsed on 182 trials, and two pairs or more were rehearsed on 194 trials. Eye movements were impossible to codify on 4 trials, due to considerable drift in the recording. Trials on which two or more pairs were rehearsed were collapsed together, to avoid producing empty cells for too many participants. In effect, 5 participants never rehearsed more than two pairs, and 12 participants never rehearsed more than three pairs. Even after collapsing together trials on which two or more pairs were rehearsed, 2 participants were dropped from the subsequent analyses because 1 had no trial on which two pairs or more were rehearsed and 1 had no trial with no pair rehearsed.

The participants' responses were scored according to a strict serial recall criterion: An item had to be in its presentation position for it to be scored as correct. The probability of correct recall as a function of the number of rehearsed pairs and serial positions is shown in Figure 1. This figure reveals a higher recall performance as the number of rehearsed pairs increased. In all the analyses, the .05 level of significance was adopted. A $3 \times 7$ repeated measures ANOVA with rehearsed pairs and serial position as factors confirmed those trends, with a significant main effect of rehearsed pairs $\left[F(2,42)=11.33, M S_{\mathrm{e}}=0.08\right]$ and of serial position $\left[F(6,126)=15.58, M S_{\mathrm{e}}=0.04\right]$, but the interaction did not reach significance $(F<1)$. Post hoc comparisons (Newman-Keuls) revealed that all three levels of rehearsed pairs significantly differed one from the other.

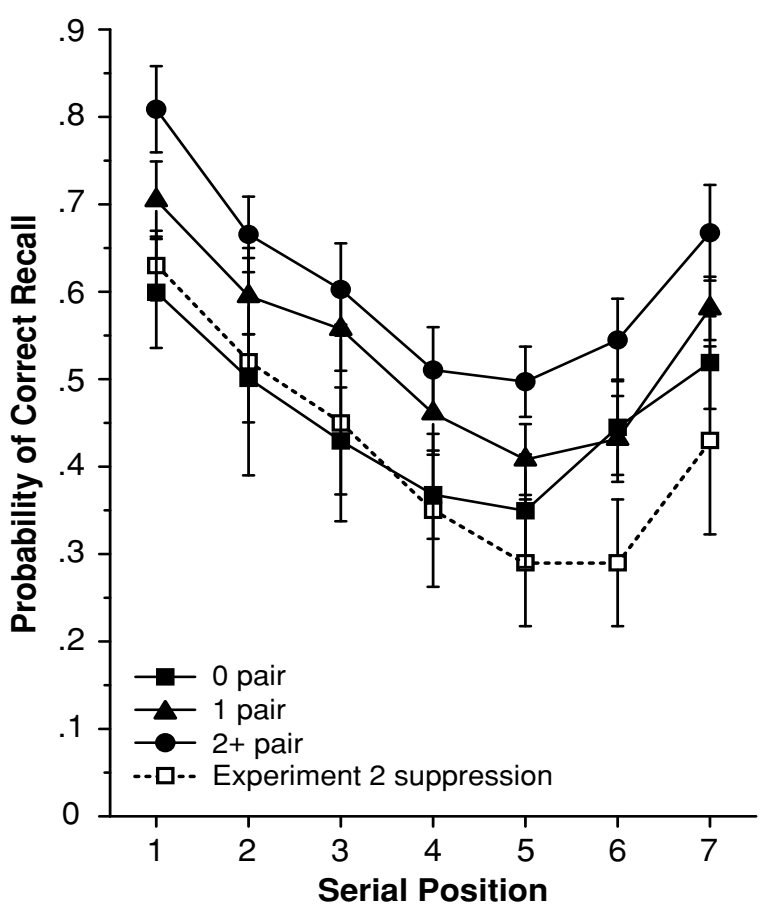

Figure 1. Mean probability of correct recall in Experiment 1 as a function of the number of rehearsed pairs and serial position and mean probability of correct recall in Experiment 2 as a function of serial position. Error bars represent the standard errors of the means.

In order to further investigate the relationship between visual-spatial rehearsal and memory performance, an additional analysis was carried out. For each list on which at least one pair of dots was rehearsed, only the dots embedded in the rehearsed pairs were kept. Consequently, for each participant, the serial position curve for the rehearsed dots was obtained by aggregating rehearsed dots from all the trials on which at least one pair was rehearsed. Performance for rehearsed dots was compared with performance for lists on which no pair of dots was rehearsed. Because not all the participants rehearsed dots on all seven positions across trials, the analysis was based on 15 of the 24 participants. As is shown in Figure 2, there was a clear and very large advantage of rehearsed dots over nonrehearsed dots of about the same magnitude at all serial positions. This trend was confirmed by a $2 \times 7$ repeated measures ANOVA with rehearsal and serial position as factors. The analysis reveals a significant main effect of rehearsal $\left[F(1,14)=16.91, M S_{\mathrm{e}}=0.111\right]$ and of serial position $\left[F(6,84)=5.31, M S_{\mathrm{e}}=0.048\right]$. The interaction did not reach significance $(F<1)$.

\section{Discussion}

The recall performance data replicate previous findings by producing the typical serial position curve with series of visual-spatial items (e.g., Jones et al., 1995; Smyth \& Scholey, 1996). A relationship was observed between the 


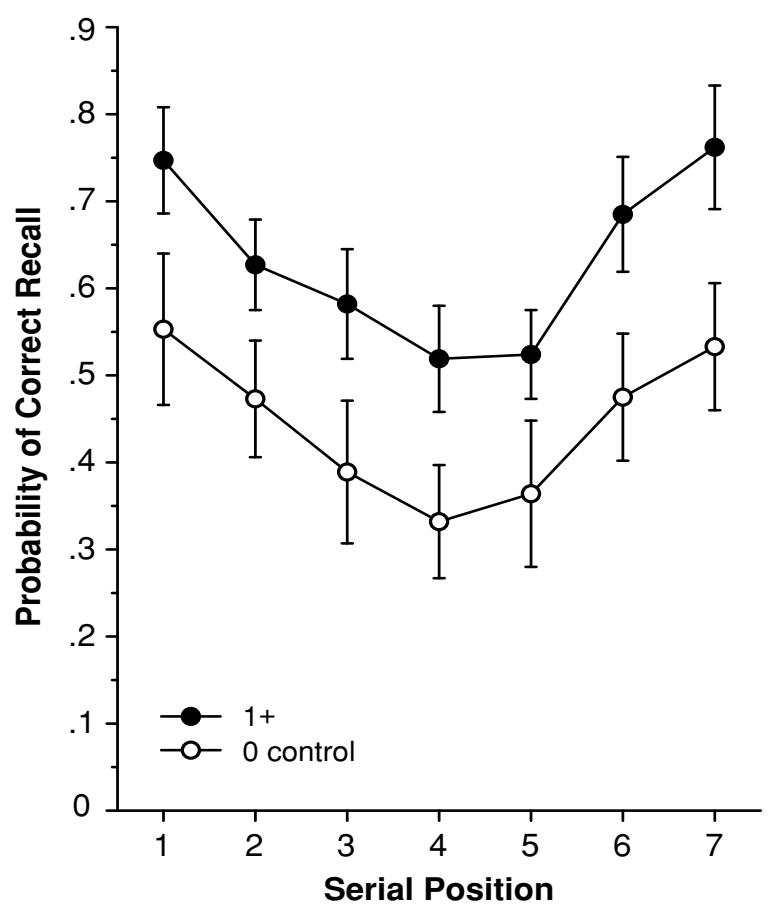

Figure 2. Experiment 1: Mean probability of correct recall with a strict serial recall criterion as a function of serial position and of whether a pair of dots had been rehearsed or not. Error bars represent the standard errors of the means.

number of rehearsed pairs and recall performance; the overall performance increased with the number of rehearsed pairs. This pattern of results suggests that the participants rehearsed the order in which the dots were initially presented by moving their eyes from one dot to the next. The use of eye movement as overt rehearsal was observable and seemed to contribute to maintaining a sequence of visualspatial items, although rehearsal did not appear to influence the shape of the serial position curves.

\section{Experiment 2}

In the second experiment, we examined the impact on recall of preventing participants from using eye movement to rehearse the TBR information during the retention interval. As in Experiment 1, all seven TBR dots were re-presented in a simultaneous fashion and remained on the screen for $10 \mathrm{sec}$ before recall. In order to prevent rehearsal, the participants were instructed to fixate in alternation on the two dots located the closest to the upper left corner of the screen. It is worth noting that these two dots were part of the initial TBR dots; there were no additional visual stimuli presented during the interval.

\section{Method}

Participants. Sixteen unpaid undergraduate students (11 women) from Université de Moncton volunteered to participate in this experiment. All the participants had normal or corrected-to-normal vision, and none of them had participated in Experiment 1.
Materials and Procedure. The materials and procedure were the same as those used in Experiment 1, except for the retention interval. The participants were instructed to systematically fixate in alternation the two dots that were nearest the top left corner.

\section{Results}

Eye movement data were first analyzed, to ensure that the participants complied with the instruction to fixate in alternation the two dots nearest the top left corner. The results revealed that per trial, the participants made between 0 and 13 fixations on the other dots, with an average of 2.2 and a median of 1 . All the trials on which there were more than 2 fixations on the other dots were removed from the analyses. With this criterion, out of the 24 trials, there were between 8 and 23 valid trials per participant, with an average of 15 . On the valid trials, the participants executed an average of $26.67(S E=0.45)$ saccades between the two dots and fixated them for $8,825 \mathrm{msec}(S E=52.44)$, which excludes the time spent performing the saccades. In the following analyses, only valid trials were considered.

As can be seen in Figure 1, recall performance in Experiment 2 was similar to that in Experiment 1 for the trials on which the participants did not rehearse any pair of dots. The pattern of results was confirmed by a $2 \times 7$ mixed design ANOVA with experiment as the between-participants factor and serial position as the repeated factor. The results revealed that recall performance was significantly lower under suppression than under the usual condition $\left[F(1,38)=9.75, M S_{\mathrm{e}}=0.151\right]$ and that there was a significant effect of serial position $[F(6,228)=38.75$, $\left.M S_{\mathrm{e}}=0.013\right]$. The interaction did not reach significance $(F=1.28, p=.27)$. The detrimental effect of suppression was further investigated by running a series of ANOVAs for each level of pairs rehearsed in Experiment 1. The first analysis was computed for the data for the condition in Experiment 1 in which no pair was rehearsed and for all the data from Experiment 2. The results revealed a main effect of serial position $\left[F(6,216)=11.55, M S_{\mathrm{e}}=0.03\right]$, but neither the main effect of experiment $(F<1)$ nor the interaction reached significance $(F=1.32)$. In the second ANOVA, the data for condition in Experiment 1 in which one pair was rehearsed was compared with all the data from Experiment 2. The results revealed a main effect of serial position $\left[F(6,216)=23.62, M S_{\mathrm{e}}=0.02\right]$ and of experiment $\left[F(1,36)=5.45, M S_{\mathrm{e}}=0.15\right]$. The interaction did not reach significance $(F<1)$. Finally, an ANOVA was performed in which the condition in Experiment 1 in which two pairs or more were rehearsed was compared with Experiment 2. The results showed a main effect of serial position $\left[F(6,216)=20.91, M S_{\mathrm{e}}=0.02\right]$ and of experiment $\left[F(1,36)=14.57, M S_{\mathrm{e}}=0.16\right]$. The interaction did not reach significance $(F<1)$.

\section{GENERAL DISCUSSION}

In the first experiment, we detected patterns of eye movement over the 10 -sec retention interval, during which the participants were free to move their eyes on the 
static re-presentation of the dots. The use of eye movement seems to be an effective rehearsal strategy, as demonstrated by the enhanced memory performance. Indeed, a consistent pattern of eye movement was revealed and appeared to modulate recall performance. The greater the quantity of visual-spatial rehearsal - indexed as the number of pairs of TBR dots that were serially fixated in the same order as in the sequence-the better the performance. Serial memory benefited from rehearsal based on eye movement, since the level of recall exceeded the level observed in Experiment 2, for which rehearsal was prevented. In Experiment 2, suppressing the use of eye movement by irrelevant saccadic eye movements reduced recall performance to the level of recall associated with the absence of rehearsal based on eye movement.

The findings of the present series of experiments demonstrate that rehearsal in the form of eye movement can take place in serial memory for sequences of visual-spatial items. Eye movement appears to represent the overt form of rehearsal, whereas covert rehearsal can be seen as taking the form of shifts of selective attention in space (e.g., Awh et al., 1998). Whether eye movement or occulomotor control can be dissociated from shifts in spatial attention remains uncertain; for some researchers, the role of eye movement in visual-spatial STM is distinct from that of attentional shifts (e.g., Lawrence et al., 2004), whereas for others there is no eye movement without a preceding reallocation of attention (e.g., Drieghe, Rayner, \& Pollatsek, 2005; see also Hoffman, 1998). For the temporary retention of item information (e.g., Awh et al., 1998), as well as for maintaining order information (e.g., Smyth, 1996), selective spatial attention seems to play a key role in covert rehearsal. There is also an indication that covert rehearsal in the form of shifts of selective attention could be supramodal and, thus, common to verbal and spatial domains (see, e.g., Zimmer et al., 2003, for a discussion). Of course, the overt form of rehearsal is intimately linked to the nature of the TBR material. Indeed, the apparent nature of overt rehearsal often mimics that of the perceived information, as exemplified by the reliance on eye movement for the retention of visual-spatial information and of inner speech for verbal information. However, that the means of rehearsal tend to differ according to the nature of TBR stimuli does not necessarily imply that different memory systems are associated with the different types of material. It may be a reification to say that there are spatial and verbal brain systems just because the nature of the stimuli afford or demand different types of rehearsal (Macken \& Jones, 2003; see also Crowder, 1993).

Our results provide clear evidence that some form of overt rehearsal involves the serial fixation of the TBR dots in the correct order of presentation. Nevertheless, the contribution of eye movement to serial memory performance does not appear to be critical. Indeed, in trials with no rehearsed pairs (Experiment 1) or when serial fixation of the TBR dots was suppressed (Experiment 2), overall performance was still relatively high, and the typical shape of the serial position curves remained. Although the observed relationship between number of rehearsed pairs and recall performance suggests that eye movement is an efficient rehearsal strategy, it seems that rehearsal based on eye movement is not the sole factor contributing to serial memory in the visual-spatial domain. That rehearsal is not essential to serial memory is not new; some researchers have provided such evidence in the verbal domain (see, e.g., Nairne, 2002).

Despite its limitations, the present study provides us with a novel approach to investigating the role of eye movement in visual-spatial rehearsal. On the basis of our results, we can argue that it is possible to study memory retrieval by tracking where participants look (see Anderson, Botthell, \& Douglass, 2004, for a discussion). Before concluding that the role of eye movement-serial fixation of the TBR items - in serial memory for visual-spatial information is restricted, further research is required. Other factors related to eye movement, such as fixation duration and fixations of items that fall outside peripheral vision, may also influence memory performance. The use of eyetracking with the dot task - the visual-spatial equivalent of the typical serial recall - has great potential for bringing new insights to the field of visual-spatial memory.

\section{REFERENCES}

Anderson, J. R., Bothell, D., \& Douglass, S. (2004). Eye movements do not reflect retrieval processes: Limits of the eye-mind hypothesis. Psychological Science, 15, 225-231.

AwH, E., \& JoNIDES, J. (2001). Overlapping mechanisms of attention and spatial working memory. Trends in Cognitive Sciences, 5, 119-126.

Awh, E., Jonides, J., \& Reuter-Lorenz, P. A. (1998). Rehearsal in spatial working memory. Journal of Experimental Psychology: Human Perception \& Performance, 24, 780-790.

BADDEley, A. D. (1986). Working memory. New York: Academic Press. Baddeley, A. D., Thomson, N., \& Buchanan, M. (1975). Word length and the structure of short term memory. Journal of Verbal Learning \& Verbal Behavior, 14, 575-589.

Crowder, R. G. (1993). Systems and principles in memory theory: Another critique of pure memory. In A. F. Collins, S. E. Gathercole, M. A. Conway, \& P. E. Morris (Eds.), Theories of memory (pp. 139161). Hillsdale, NJ: Erlbaum.

Drieghe, D., Rayner, K., \& Pollatsek, A. (2005). Eye movements and word skipping during reading revisited. Journal of Experimental Psychology: Human Perception \& Performance, 31, 954-959.

Farmer, E. W., Berman, J. V. F., \& Fletcher, Y. L. (1986). Evidence for a visuo-spatial scratch-pad in working memory. Quarterly Journal of Experimental Psychology, 38A, 675-688.

FARRELl, S., \& LeWANDOWSKY, S. (2002). An endogenous distributed model of ordering in serial recall. Psychonomic Bulletin \& Review, 9, 59-79.

Hoffman, J. E. (1998). Visual attention and eye movements. In H. Pashler (Ed.), Attention (pp. 119-153). Hove, U.K.: Taylor \& Francis.

Jones, D., Farrand, P., Stuart, G., \& Morris, N. (1995). Functional equivalence of verbal and spatial information in serial short-term memory. Journal of Experimental Psychology: Learning, Memory, \& Cognition, 21, 1008-1018.

Lawrence, B. M., Myerson, J., \& Abrams, R. A. (2004). Interference with spatial working memory: An eye movement is more than a shift of attention. Psychonomic Bulletin \& Review, 11, 488-494.

Lawrence, B. M., Myerson, J., Oonk, H. M., \& Abrams, R. A. (2001). The effects of eye and limb movements on working memory. Memory, 9, 433-444.

Logie, R. H. (1995). Visuo-spatial working memory. Hillsdale, NJ: Erlbaum.

Longoni, A. M., Richardson, J. T. E., \& Aiello, A. (1993). Articulatory rehearsal and phonological storage in working memory. Memory \& Cognition, 21, 11-22. 
MACKEN, W. J., \& JONES, D. M. (2003). Reification of phonological storage. Quarterly Journal of Experimental Psychology, 56A, 1279-1288.

Meiser, T., \& Klauer, K. C. (1999). Working memory and changingstate hypothesis. Journal of Experimental Psychology: Learning, Memory, \& Cognition, 25, 1272-1299.

NAIRNE, J. S. (2002). Remembering over the short-term: The case against the standard model. Annual Review of Psychology, 53, 53-81.

Pearson, D. G., \& Sahraie, A. (2003). Oculomotor control and the maintenance of spatially and temporally distributed events in visuospatial working memory. Quarterly Journal of Experimental Psychology, 56A, 1089-1111.

Postle, B. R., Awh, E., Jonides, J., Smith, E. E., \& D’Esposito, M. (2004). The where and how of attention-based rehearsal in spatial working memory. Cognitive Brain Research, 20, 194-205.

Rundus, D., \& AtKinson, R. C. (1970). Rehearsal processes in free recall: A procedure for direct observation. Journal of Verbal Learning \& Verbal Behavior, 9, 99-105.

SCHWEICKERT, R., \& BORUFF, B. (1986). Short-term memory capacity: Magic number or magic spell? Journal of Experimental Psychology: Learning, Memory, \& Cognition, 12, 419-425.

Sмүтн, M. M. (1996). Interference with rehearsal in spatial working memory in the absence of eye movements. Quarterly Journal of Experimental Psychology, 49A, 940-949.

Smyth, M. M., \& Scholey, K. A. (1994). Interference in immediate spatial memory. Memory \& Cognition, 22, 1-13.

Smyth, M. M., \& Scholey, K. A. (1996). Serial order in spatial immediate memory. Quarterly Journal of Experimental Psychology, 49A, 159-177.

Zelinsky, G. J., \& LoschKy, L. C. (2005). Eye movements serialize memory for objects in scenes. Perception \& Psychophysics, 67, 676690.
Zimmer, H. D., Speiser, H. R., \& SEIDLER, B. (2003). Spatio-temporal working-memory and short-term object-location tasks use different memory mechanisms. Acta Psychologica, 114, 41-65.

\section{NOTE}

1. There are other ways to look at eye movement data in relation to serial memory performance. For instance, one is to compute the longest sequence of dots rehearsed in the correct order, instead of the number of adjacent dot pairs rehearsed in the correct order. Both performance measures produce very similar patterns of results. The number of pairs was preferred here because there was a slightly more similar number of observations across levels (number of rehearsed pairs) than that observed with the longest sequence criterion (length of the longest rehearsed sequence). The number of saccades in the correct order between adjacent dots was also computed. This measure was not chosen, because it confounds repeated rehearsal and number of items rehearsed. Indeed, a score of 10 could be obtained either if a given dot pair was rehearsed 10 times or if five out of the six adjacent dot pairs were rehearsed twice each. Finally, the number of adjacent dot pairs rehearsed and the number of saccades between adjacent dot pairs could also be computed, irrespective of the order. In other words, if there was a saccade between the fifth and the sixth dots, one point would be given if the saccade was from the fifth to the sixth - as was used with the indexes reported above - as well as if the saccade was in the reverse order. Once more, the pattern of results obtained with such a criterion is very similar to the one reported with the measures presented above, although the relationship with recall performance is weaker than if only saccades in the correct order are considered.

(Manuscript received April 7, 2005; revision accepted for publication October 12, 2005.) 